\title{
Perinatal transfer of genetic information: developing an algorithm for reporting cystic fibrosis prenatal test results to the newborn screening program
}

\author{
Ellen S. Regalado, $M S^{1}$, Elinor Langfelder-Schwind, $M S^{2,3}$, Andrew D. Corwin, $M D^{2,3}$, and Kenneth A. Pass, PhD
}

\begin{abstract}
Purpose: This study explored the feasibility of statewide reporting of cystic fibrosis fetal diagnostic testing results to the newborn screening program through the birth hospital. Methods: We evaluated trends in offering and documenting cystic fibrosis carrier screening among prenatal care providers through a survey of 100 medical records of patients who gave birth at St. Vincent's Hospital Manhattan. The hospital's protocol for reporting human immunodeficiency virus testing history to the state program was delineated and adapted in developing an algorithm for cystic fibrosis. Feedback from hospital staff with regard to data transcription and the prospect of transferring cystic fibrosis prenatal information was obtained. Results: Of 98 patients who had prenatal records made available to the birth hospital, $62 \%$ had cystic fibrosis carrier screening, $14 \%$ declined screening, and $24 \%$ had no documentation of their screening history. The hospital staff viewed the transcription of information as relatively simple; however, missing information is a common occurrence that delays the process and results in incomplete data transfer. Conclusions: Perinatal transfer of cystic fibrosis prenatal information modeled on the system used for reporting human immunodeficiency virus testing history is feasible. However, it will require standardized reporting of cystic fibrosis screening and testing history on the mother's prenatal records among prenatal care providers. Genet Med 2008:10(11):805-810.
\end{abstract}

Key Words: Cystic fibrosis, carrier screening, fetal diagnostic testing, newborn screening

Cystic fibrosis (CF) is an autosomal recessive condition that usually presents in infancy as failure to thrive because of pancreatic insufficiency and chronic lung problems. ${ }^{1}$ More than 1500 mutations have been identified on the cystic fibrosis transmembrane regulator (CFTR) gene ${ }^{2}$ resulting in a defective electrolyte transport in the apical membrane epithelial cells of the lungs, pancreas, intestine, liver, sweat glands, and the male reproductive tract. ${ }^{3}$ The condition is most common among non-Hispanic white and Ashkenazi Jewish populations with birth prevalence estimates of 1:3300 and 1:2270, respectively, and less common in Hispanic whites $(1: 13,500)$, African Americans (1:15,100), and Asian Americans $(1: 35,100) .{ }^{4}$

In 2001, the American College of Obstetricians and Gynecologists (ACOG) recommended that CF prenatal carrier screening be offered to individuals of white and Ashkenazi Jewish ethnicity who are at higher risk for $\mathrm{CF}$, and be made known to other ethnicities as part of routine prenatal and preconception care. ${ }^{5} \mathrm{Cou}-$ ples who are carriers may choose prenatal diagnostic testing

From ${ }^{1}$ Sarah Lawrence College, Bronxville, New York; ${ }^{2}$ St. Vincent Catholic Medical Center, St. Vincent's Hospital Manhattan, New York, New York; ${ }^{3}$ New York Medical College, Valhalla, New York; ${ }^{4}$ New York State Department of Health, Albany, New York.

Kenneth A. Pass, PhD, Wadsworth Center, New York State Department of Health, P.O. Box 509, Albany, NY 12201-0509. E-mail: kpass@wadsworth.org

Disclosure: The authors declare no conflict of interest.

Submitted for publication April 9, 2008.

Accepted for publication August 6, 2008.

DOI: $10.1097 /$ GIM.0b013e31818a3107 to determine whether a fetus has CF. Although the American College of Medical Genetics recommended a panel of 25 CFTR mutations for routine diagnostic and carrier screening, ${ }^{6}$ expanded mutation panels and sequencing analysis are offered by several laboratories. The clinical sensitivities of CF prenatal screening using the recommended panel were estimated to be $88.6 \%, 71.9 \%, 51.7 \%, 41.6 \%$, and $23.4 \%$ for Ashkenazi Jewish, non-Hispanic white, Hispanic white, African American, and Asian American couples, respectively. ${ }^{4}$

Universal newborn screening for CF was implemented in New York State (NYS) in 2002 and is fully implemented in 44 other states. ${ }^{7}$ The NYS newborn screening (NBS) program uses an IRT/DNA algorithm, which begins with an immunoreactive trypsinogen (IRT) test on dried blood spots followed by DNA analysis of 40 CFTR mutations on specimens with an elevated IRT test. Newborns who have an elevated IRT and at least one detected mutation are reported as screen positive, and are referred to a diagnostic center for sweat chloride testing and appropriate medical management. ${ }^{8}$ Newborns with two disease causing mutations are considered to have $\mathrm{CF}$ regardless of the sweat chloride test results. ${ }^{9}$

The NYS NBS protocol for CF has a sensitivity of $98 \%$ and specificity of $99.5 \% .{ }^{10}$ In its first 2.5 years of implementation, Giusti et al. ${ }^{10}$ reported that the state program failed to identify at least two affected newborns who were reported instead as screen negative. False-negative results can lead to delayed implementation of CF treatment protocols for an affected new- 
born, and cause confusion and false reassurance among parents and primary care providers. When a newborn is previously identified as having $\mathrm{CF}$ through prenatal diagnostic testing, improving the communication between the prenatal and newborn CF screening programs can potentially enhance the newborn screening algorithm resulting in faster diagnosis, more immediate referral, and possibly reduced costs. Coordination between prenatal screening and newborn screening programs becomes essential as more and more genetic conditions are screened and tested in the prenatal and newborn periods.

In looking at ways to report prenatal CF screening history and fetal diagnostic test results to the state NBS program, the birth hospital seems to be the appropriate venue for collecting this information and forwarding it to the state laboratory. Currently, the hospital of birth is responsible for collecting the blood specimen and sending it, along with demographic and other information, to the state laboratory. Birth hospitals in NYS already report maternal human immunodeficiency virus (HIV) testing history and status, and any expedited HIV testing performed during labor on patients who earlier declined testing or otherwise required immediate repeat testing, or on the newborn on the NBS blood collection form as part of the State Maternal-Pediatric HIV Prevention and Care Program. ${ }^{11}$ The algorithm for reporting HIV testing history and results to the NYS NBS program is a potential model for transferring prenatal CF carrier screening history and fetal diagnostic testing results to the state program.

This study assessed the feasibility of transferring CF carrier screening history and fetal diagnostic testing results to the state newborn screening program by examining the practices of documenting this information in the mother's medical records among prenatal providers affiliated with one urban hospital serving an ethnically diverse patient population. Current hospital protocols in completing the NBS blood collection form and transferring maternal HIV testing history from the maternal chart to the form were delineated. In addition, feedback from hospital staff with regard to completing the NBS form and the prospect of the additional task of transcribing CF carrier screening and fetal diagnostic testing results was obtained. Using HIV as a model, an adapted algorithm for achieving transfer of CF carrier screening history and fetal diagnostic testing results to the newborn screening program is proposed.

\section{MATERIALS AND METHODS}

The research protocol was approved by the Institutional Review Boards of the New York State Department of Health and Saint Vincent Catholic Medical Centers (SVCMC). The study was conducted at the SVCMC-St. Vincent's Hospital Manhat$\tan$ (SVHM), which provides prenatal care at its Resident O'Toole and Chinatown clinics, and obstetrical services to patients referred through these clinics as well as private offices in the metropolitan New York area.

The hospital's procedure for reporting maternal and expedited HIV testing history to the state newborn screening pro- gram was first examined through interviews with the staff of the labor and delivery, and postpartum units. The nurses and clerks in the labor and delivery, and postpartum units of SVHM were also interviewed by one of the authors (E.R.) using a standard set of questions. The participants were asked about their assessment of the current procedure of completing the form, problems they have encountered in completing the form, and their suggestions for improving the current procedure of transcribing information for the state lab. Their awareness of cystic fibrosis prenatal carrier screening and how often they have encountered reports of CF screening or testing while going through prenatal records were assessed. Specifically, they were asked about the ease or difficulty of locating CF screening history and diagnostic testing results in the mother's records, their ability to accurately transfer this information from the medical records onto the NBS sample collection form, and their suggestions in terms of education and training if such data transfer is to be implemented by the state program.

A survey of 100 consecutive labor and delivery medical charts of patients who gave birth at SVHM before December 31,2005 was conducted. Site of prenatal care, presence or absence of family history of $\mathrm{CF}$, presence or absence of documentation of CF screening consent/dissent, CF carrier screening results for patient and reproductive partner if available, and patient's ethnicity as noted by the prenatal care provider or SVHM were extracted from the patient's medical records. Frequencies were calculated and $\chi^{2}$ test of association was performed when possible.

\section{RESULTS}

\section{SVCMC-SVHM protocol for reporting HIV testing history and status to the NBS program}

The protocol for reporting maternal HIV testing history and results at SVCMC-SVHM complies with the NYS Department of Health Maternal-Pediatric HIV Prevention and Care Program, which requires maternal HIV testing done during or before the pregnancy and expedited HIV testing status at time of birth to be reported on the NBS blood collection form. To improve compliance, a copy of a quarterly audit highlighting absent HIV reporting is sent to the chief administrative officer of each hospital. Although the goal is for all pregnant women to be tested before labor, expedited testing is offered in the labor and delivery setting if the mother has not been tested before labor, if there is no documentation of her tests, or if her history indicates a reason that she be tested again. In these circumstances, expedited testing is performed on the mother or on the newborn if she declined testing.

Patients who give birth at SVCMC-SVHM present to the hospital with or without prenatal care history. Patients who had prenatal care by a private physician or a certified nurse/ midwife have copies of their prenatal records sent to the hospital at about their 36th week of gestation. Likewise, prenatal records of patients seen at the Chinatown Clinic and the Resident O'Toole clinic are forwarded to the hospital at this time. There are a small number of patients who present to the hos- 
pital as emergency cases and may have had no prior prenatal care during the pregnancy or had prenatal care by an unaffiliated provider. As such, their prenatal records are unavailable at the time of their arrival at the labor room.

In the ideal circumstance, the prenatal records are available to the labor room staff and contain medical information and prenatal laboratory data including documentation of HIV testing performed during the present gestation. These prenatal records become part of the labor and delivery chart of the mother. On admission, the data are checked and a decision is made regarding the patient's HIV status and the need for expedited testing and/or prophylactic antiviral medication.

After birth, the attending physician and/or the labor room staff transcribes information about HIV testing done before labor along with any in-hospital expedited testing on to the NYS Maternal-Pediatric HIV Prevention and Care Program Test History and Assessment form. Data are recorded according to a two letter classification, of letters $A$ to $D$ and $E$ to G, where A means mother tested HIV-negative during this pregnancy, B means mother tested HIV-positive during or before this pregnancy, $\mathrm{C}$ means mother was not tested during this pregnancy, D means test history is unknown or not documented, E means mother consented to and received expedited testing, F means newborn was tested, and G means expedited testing is not needed because mother was tested previously. The form has three copies, which are separated and filed with one copy each in the hospital chart of the mother and the baby, and one copy forwarded to the hospital's HIV follow-up officer.

The ward clerk or nurse at the labor and delivery setting completes the NBS blood collection form, and transfers the HIV testing history and status using the same letter classifications as above. Normally, the clerk at the labor room completes the form when all the necessary information is found in the charts. Otherwise, the charts and the NBS form move with the newborn to the postpartum and nursery units, and any missing information is added to the NBS form as it is obtained. The phlebotomist or the pediatric resident then collects the blood specimen from the newborn and the NBS blood collection form is sent to the state newborn screening program.

\section{Documentation of CF carrier screening and fetal diagnostic testing history on the mother's prenatal records}

The distribution in terms of ethnicity and site of prenatal care of the 100 patients whose records were included in the survey is shown in Table 1. SVHM Resident O'Toole clinic saw a fairly diverse patient population whereas SVHM Chinatown clinic had a $100 \%$ Asian patient population. Private prenatal clinics saw mainly white patients $(70 \%)$ as well as patients of Asian, Hispanic, and other ethnicities.

Of the 100 maternal charts surveyed, 98 had a record of the mother's prenatal care history. One patient who was seen by a private care provider and another patient who was seen at the SVHM Resident O'Toole clinic did not have a record of their prenatal care history. CF carrier screening history of the patient, which includes patient consent or dissent and the test
Table 1

Distribution of survey population according to ethnicity and site of prenatal care

\begin{tabular}{lccc}
\hline & \multicolumn{3}{c}{ Site of prenatal care } \\
\cline { 2 - 4 } Ethnicity $n(\%)$ & $\begin{array}{c}\text { Private } \\
\text { clinics }\end{array}$ & $\begin{array}{c}\text { SVHM Resident } \\
\text { O’Toole clinic }\end{array}$ & $\begin{array}{c}\text { SVHM Chinatown } \\
\text { clinic }\end{array}$ \\
\hline White & $35(70)$ & $4(16)$ & $0(0)$ \\
African American & $0(0)$ & $6(24)$ & $0(0)$ \\
Hispanic & $5(10)$ & $7(28)$ & $0(0)$ \\
Asian & $5(10)$ & $3(12)$ & $25(100)$ \\
Other & $5(10)$ & $5(20)$ & $0(0)$ \\
Total & 50 & 25 & 25 \\
\hline
\end{tabular}

results if CF carrier screening was done, was reported in $76 \%$ of the charts. Eighty-seven percent of patients who were seen at the Resident O'Toole clinic and $80 \%$ of patients who were seen at the Chinatown clinic had a record of their CF carrier screening history. In contrast, only $69 \%$ of the patients who were seen by private prenatal care providers had a record of their CF carrier screening history. There is no significant difference in the reporting of $\mathrm{CF}$ carrier screening history between the O'Toole and Chinatown clinics, $X^{2}(1, N=49)=0.105, P>$ 0.05 although there are descriptive differences that favor the hospital-based prenatal care providers over private prenatal care providers in reporting CF carrier screening history. Two patients' charts contained a record of their partner's CF carrier screening results.

Of the 75 patients with documentation of their CF carrier screening history, $81 \%$ had CF carrier screening, while $19 \%$ declined CF carrier screening (Table 2). Dissent was documented in prenatal records of two patients who were seen at the Resident O'Toole clinic and 12 patients who were seen at

\section{Table 2}

Documentation of consent/dissent and uptake of CF screening among sites of prenatal care and patient ethnicities $(n=98)$

\begin{tabular}{lccc}
\hline & $\begin{array}{c}\text { Consented } \\
\text { to screening }\end{array}$ & $\begin{array}{c}\text { Declined } \\
\text { screening }\end{array}$ & Undocumented \\
\hline $\begin{array}{l}\text { Site of prenatal care, } n(\%) \\
\text { SVHM O’Toole clinic }\end{array}$ & $19(79)$ & $2(8)$ & $3(13)$ \\
$\begin{array}{l}\text { SVHM Chinatown } \\
\text { clinic }\end{array}$ & $8(32)$ & $12(48)$ & $5(20)$ \\
$\quad$ Private clinics & $34(69)$ & $0(0)$ & $15(31)$ \\
Ethnicity, $n(\%)$ & & & \\
$\quad$ White & $27(71)$ & $1(3)$ & $10(26)$ \\
African American & $5(83)$ & $0(0)$ & $1(17)$ \\
Hispanic & $10(83)$ & $0(0)$ & $2(17)$ \\
Asian & $12(37.5)$ & $12(37.5)$ & $8(25)$ \\
$\quad$ Other & $7(70)$ & $1(10)$ & $2(20)$ \\
Total & $61(62)$ & $14(14)$ & $23(24)$ \\
\hline
\end{tabular}


the Chinatown clinic. Documentation that CF carrier screening was offered and/or the patient declined was not observed in prenatal records of patients who were seen by private prenatal care providers.

Table 2 also shows the number of women from different ethnic groups who consented to and declined CF carrier screening. Although none of the patients had a family history of CF, uptake of CF carrier screening appears to differ significantly among ethnic groups. Fifty percent of Asian patients consented to CF screening whereas $88 \%-100 \%$ of Caucasian, African American, Hispanic patients, and patients of other ethnicities consented to CF carrier screening.

Prenatal care providers noted CF screening test results, i.e., "CF negative," on a standardized prenatal form (51\%), or provided a hard copy of the laboratory report with or without a written note on the prenatal form (49\%). Among the private prenatal care providers, $85 \%$ noted the CF screening test results on the prenatal form whereas $15 \%$ provided a copy of the laboratory report with or without a written note on the prenatal form. On the other hand, the majority of hospital prenatal care providers (100\% in Resident O'Toole clinic and $75 \%$ in Chinatown clinic) provided a copy of the laboratory report with or without a written note on the prenatal form. The method of reporting CF screening test results is not significantly different between the Resident O'Toole and Chinatown clinics, $X^{2}(1, N=27)=2.132, P>0.05$. However, it is significantly different between private care providers and the hospital clinics, $X^{2}(1, N=61)=33.477, P<0.001$.

None of the 98 prenatal records contained documentation of a positive CF carrier screening result, and therefore none of the charts had results of fetal diagnostic testing for CF. Practices of documenting CF fetal diagnostic testing and results among prenatal care providers were not assessed.

\section{Interviews with hospital staff}

Two nurses and a clerk at the labor and delivery unit and two nurses at the postpartum unit agreed to be interviewed to assess their roles in the newborn screening process and their responses toward the additional task of transcribing CF prenatal information. In this hospital setting, the clerk searches for information in the maternal and newborn charts, and transfers needed information to the NBS blood collection form. Three of the nurses interviewed replied that their role in the process of completing the NBS form is minimal. They assist the clerk in contacting the patient's prenatal care provider when the information is not available in the charts and become more involved in the absence of the clerk. One nurse reported that she is responsible for ensuring that the NBS blood collection form has the complete information and valid blood specimen before it is sent to the state lab.

Despite the small sample size $(n=5)$, the responses were consistent on key issues: (1) the current process for completing the HIV and NBS screening forms are viewed as relatively simple (avg. 1.6 on a 5-point Likert scale with 5 being most difficult), and (2) interviewees reported that it would be simple to locate the information if appropriately placed in the chart (avg.
1.2 on a 5-point Likert scale with 5 being most difficult). However, three of five interviewees acknowledged that although the process seems relatively simple, it requires some effort and careful attention because of the amount of information required. The most significant problems anticipated were absence of information in the record and potential for human error in transcription. Although one interviewee indicated that the system is working well and another could not suggest ways to improve it, the others cited that the prenatal care providers could do better to ensure that the patient's prenatal record is complete when forwarded to the birth hospital. Most of the interviewees are aware of CF prenatal testing. Two nurses have seen CF test reports while going through the patient medical charts although they recognize that currently there is yet no consistency among prenatal providers in reporting this information. They anticipate that training will be provided in transcribing this information to the newborn screening form but simply view it as one more information to locate and transfer to the form.

\section{DISCUSSION}

In assessing the feasibility of a statewide reporting of CF carrier screening history and fetal diagnostic testing results to the newborn screening program, we examined if this information is being reported routinely on the mother's prenatal records. In summary, $2 \%$ of patients did not have a record of their prenatal care history and $23 \%$ of patients did not have documentation of their CF carrier screening history.

With this patient population, $81 \%$ of those who had documentation of their CF screening history chose to be screened for CF. More than $88 \%$ of white, African American, Hispanic, and patients of other ethnicities were screened for CF while lower uptake was observed among Asian patients (50\%) as might be expected because of the lower detection rate of CF screening in this population. None of the patients were identified as carrier of a CFTR mutation. Thus, the practice of documenting $\mathrm{CF}$ fetal diagnostic testing was not assessed.

Our results showed that there is no standardized practice among prenatal care providers of documenting whether or not $\mathrm{CF}$ carrier screening was offered, and the resulting consent or dissent. Currently, prenatal care providers use different methods of documenting CF screening test results. Although some included a laboratory report in the prenatal record, others noted the results on a standardized prenatal form. Hospital prenatal care providers following institutional protocols were more consistent in documenting CF carrier screening history compared with private prenatal care providers. Routines for offering and documenting CF carrier screening likely differ among practice sites. At the SVHM-Resident O'Toole clinic, CF screening is offered at the first prenatal visit. Linguistically appropriate written materials, developed in collaboration between the Obstetrics and Genetics staff, are distributed to each patient as well. These materials were adapted and translated for the SVHM-Chinatown clinic, which is staffed by Chinesespeaking medical professionals. The CF carrier screening prac- 
tices of affiliated private physicians are not known and were not assessed as part of this study.

A survey of practice patterns of obstetrician-gynecologists regarding $\mathrm{CF}$ preconception and prenatal screening 2 years after the ACOG guidelines were released showed that only $66 \%$ offered screening to all prenatal patients. ${ }^{12}$ Although the ACOG guidelines advise practitioners to obtain written informed consent to request or decline screening, prenatal care providers may not necessarily document on the patient's records that CF prenatal screening was not offered. Absence of documentation of CF carrier screening history may mean CF carrier screening was not offered, the patient declined carrier screening, or perhaps that the patient consented and the results were negative. Lack of documentation of patient's screening history may be more common in clinical settings where patients are predominantly from ethnic groups at lower risk for $\mathrm{CF}$, such as African American, Hispanic, or Asian patients for whom carrier screening may be made available only on request. ${ }^{5,13}$ However in our study, which included a clinical setting where the patients are predominantly at lower risk for CF (Chinatown clinic), a majority of patients have documentation of their carrier screening history primarily because institutional protocols requiring hospital prenatal care providers to document this information are in place. Of note, New York State has strict guidelines for obtaining and documenting informed consent before ordering genetic testing on a patient for diagnostic purposes ${ }^{14}$; it is not clear whether the obstetrical community at large is aware of these policies or whether other states would have similar patterns of documentation.

Practice patterns among prenatal care providers as well as the site of prenatal care will likely determine the success of statewide reporting of CF prenatal information to the NBS program. Missing documentation of CF carrier screening history for a significant number of patients and incomplete data transfer will likely be problematic for the birth hospital completing the NBS form and the newborn screening officer who has to track down this information.

The mechanism for collecting and reporting HIV testing history and status of the mother and newborn to the newborn screening program appears to be well established across the state. Based on data extracted from the NBS blood collection form, the NYS Department of Health reported a steady increase in prenatal HIV testing rate from $<50 \%$ in 1997 to more than $93 \%$ in $2001 . .^{15}$ The system used for reporting HIV information to the state program offers a suitable model for developing a similar algorithm for cystic fibrosis. We described the experience of a metropolitan birth hospital in reporting this information on the NBS blood collection form and showed some of the challenges associated with the process that should inform the implementation of a statewide reporting of CF diagnostic testing results to the state laboratory.

In practice, the use of a two letter classification in reporting HIV testing history and status creates confusion because it does not cover all possible testing circumstances and certain letter combinations have been found to be conflicting. It is an additional step in the transcription process that can be prone to mistakes. Data that becomes available after the three-copy Maternal-Pediatric HIV Prevention and Care Program Test History and Assessment form is separated may not be transcribed on all three copies and the NBS blood collection form resulting in inconsistent information reported on these forms.

Currently, information is manually transcribed from the mother's and newborn's medical records to the NBS blood collection form. Thus the quality of information submitted to the state lab essentially depends on the accuracy of transcription by hospital staff. While hospital staff viewed the transcription process as relatively simple, they recognized the potential for human error. They also cited missing prenatal information as a recurring problem that slows down the process of transcription, consumes staff time, and results in incomplete data transfer. During holidays and long weekends or when the prenatal care provider is unable to send the information promptly, this information may not be retrieved in a timely manner and thus not reported to the newborn screening program. It will then be the task of the newborn screening program's follow-up officer to request this information from the birth hospital or the prenatal care provider.

In contrast to reporting HIV information, which requires multiple testing circumstances to be summarized and recorded on the NBS form as well as the Maternal-Pediatric HIV Prevention and Care Program Test History and Assessment form, reporting CF screening history and fetal diagnostic testing results is expected to be more straightforward. The hospital staff reviews the CF carrier screening history of the patient and her
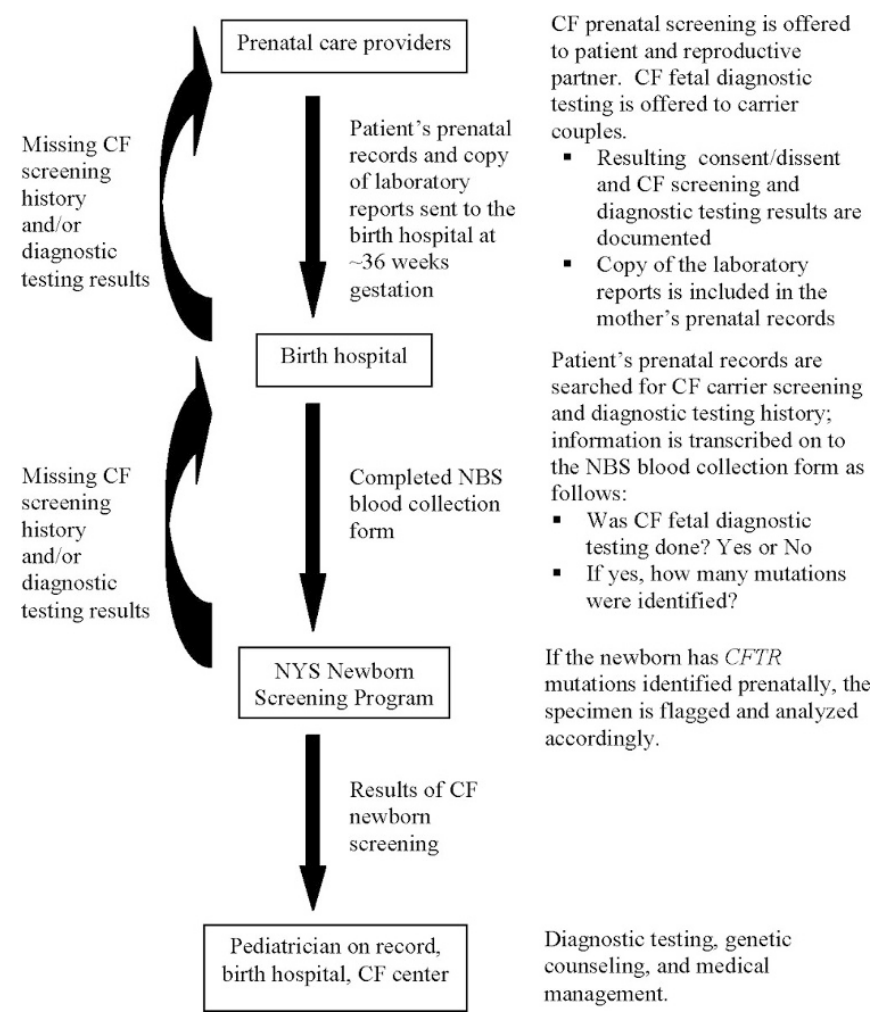

CF prenatal screening is offered to patient and reproductive partner. CF fetal diagnostic testing is offered to carrier couples.

- Resulting consent/dissent and CF screening and diagnostic testing results are documented

- Copy of the laboratory reports is included in the mother's prenatal records

Patient's prenatal records are searched for CF carrier screening and diagnostic testing history; information is transcribed on to the NBS blood collection form as follows:

- Was CF fetal diagnostic testing done? Yes or No

- If yes, how many mutations were identified?

If the newborn has CFTR mutations identified prenatally, the specimen is flagged and analyzed accordingly.

Fig. 1. Proposed algorithm for reporting CF prenatal testing results to the NYS newborn screening program. 
reproductive partner, locates any fetal diagnostic testing results, and transcribes this information directly on the NBS form by answering two questions: "Was CF fetal diagnostic testing done?" and "If so, how many mutations were identified?" Adding these two fields of information to the current NBS form would be sufficient to identify newborns who have been diagnosed with CF prenatally. Although inclusion of the parents' carrier test results would be helpful for counseling purposes on those infants who screen positive but do not have $\mathrm{CF}$, it is not feasible at this time given the very limited space of the form. However, reporting this information could be considered in the future if the State implements electronic transfer of NBS information.

Using the protocol for reporting maternal HIV testing history to the NBS program as a model, an algorithm for reporting CF prenatal information to the program is proposed (Fig. 1). In implementing reporting of CF prenatal information, the hospital staff will need to be trained to understand the algorithm of CF carrier screening and fetal diagnostic testing and annotations used by prenatal care providers and testing laboratories, and locate CF carrier screening history and fetal diagnostic testing results in the prenatal records.

When the CF-affected status of the newborn is known prenatally, coordination of information between the CF prenatal and newborn screening programs ensures that affected newborns are identified by either or both programs. Perinatal transfer of CF prenatal diagnostic testing results to the newborn screening program that is modeled on the system used by NYS for reporting HIV information is feasible. However, our results showed that CF carrier screening history is not documented for a significant number of patients reflecting a current lack of consistency among prenatal care providers in documenting this information, the consent process, and possibly in offering CF carrier screening. Achieving statewide reporting of CF prenatal information to the newborn screening program will require instituting standardized reporting of this information on the maternal record that includes proper documentation of informed consent or dissent and CF carrier screening and diagnostic testing results among prenatal care providers. Birth hospitals will also need to establish institutional protocols in collecting this data as well as train hospital staff to accurately transfer this information to the state newborn screening program.

\section{ACKNOWLEDGMENTS}

This study was supported by cooperative agreement U10/ CCU224489-01 ("Improving the Quality of Genetic Testing and Assuring Its Appropriate Integration into Clinical and Public Health Practice") from the Centers for Disease Control and Prevention to the New York State Newborn Screening Program.

The authors thank Dr. Ira Lubin for his suggestions and review of the manuscript.

\section{References}

1. Kaye CI; the Committee on Genetics. Newborn screening fact sheets. Pediatrics 2006;116:e934-e963.

2. Cystic Fibrosis Mutation Database Toronto: The Hospital for Sick Children, Genetics and Genomic Biology, 2007. Available at: http://www.genet.sickkids.on.ca/cftr/ StatisticsPage.html.

3. Welsh MJ, Ramsey BW, Accurso F, Cutting GR. Cystic fibrosis. In: Scriver CR, Beaudet AL, Valle D, Sly WS, editors. The metabolic and molecular bases of inherited disease, 8th ed. New York: McGraw Hill, 2001:5121-5188.

4. Palomaki GE, FitzSimmons SC, Haddow JE. Clinical sensitivity of prenatal screening for cystic fibrosis via CFTR carrier testing in a United States panethnic population. Genet Med 2004;6:405-414.

5. American College of Obstetricians and Gynecologists, American College of Medical Genetics, National Institutes of Health. Preconception and prenatal carrier screening for cystic fibrosis: clinical and laboratory guidelines. Washington, DC: American College of Obstetricians and Gynecologists, 2001.

6. Watson MS, Cutting GR, Desnick RJ, et al. Cystic fibrosis population carrier screening: 2004 revision of American College of Medical Genetics mutation panel. Genet Med 2004;6:387-391.

7. National Newborn Screening and Genetics Resource Center. National Newborn Screening Status Report. Available at: http://genes-r-us.uthscsa.edu/nbsdisorders. pdf. Accessed August 3, 2008.

8. New York State Department of Health. Newborn Screening in New York State: A Guide for Health Professionals. Albany: NYS Department of Health, 2003.

9. Rosenstein BJ, Cutting GR. The diagnosis of cystic fibrosis: a consensus statement. Cystic Fibrosis Foundation Consensus Panel. J Pediatr 1998;132:589-595.

10. Giusti R, Badgwell A, Iglesias AD; the New York State Cystic Fibrosis Newborn Screening Consortium. New York State Cystic Fibrosis Consortium: the first 2.5 years of experience with cystic fibrosis newborn screening in an ethnically diverse population. Pediatrics 2007;119:460-467.

11. New York State Department of Health. Annual HIV Summary Report 1999 Edition. Available at: http://www.health.state.ny.us/diseases/aids/newborn/annualreports/ intro99.html. Accessed November 27, 2007.

12. Morgan MA, Driscoll DA, Mennuti MT, Schulkin J. Practice patterns of obstetrician-gynecologists regarding preconception and prenatal screening for cystic fibrosis. Genet Med 2004;6:450-455.

13. Farrell PM, Fost N. Prenatal screening for cystic fibrosis: where are we now? J Pediatr 2002;141:758-763.

14. New York State Task Force on Life and the Law. Genetic testing and screening in the age of genomic medicine. Available at: http://www.health.state.ny.us/nysdoh/ taskfce/screening.htm. Accessed March 18, 2008.

15. New York State Department of Health. Annual HIV Summary Report 2001 Edition. Available at: http://www.health.state.ny.us/diseases/aids/newborn/annualreports/ intro2001.html. Accessed April 28, 2007. 